\title{
A PROBABILITY BOUND FOR INTEGRALS WITH RESPECT TO STOCHASTIC PROCESSES WITH INDEPENDENT INCREMENTS ${ }^{1}$
}

D. L. HANSON AND L. H. KOOPMANS

1. Introduction. The purpose of this paper is to establish the following theorem.

TheOREM. Let $X=\left\{X_{t}:-\infty<t<\infty\right\}$ be a real-valued stochastic process with independent increments. Let $\mu$ be a $\sigma$-finite measure on the real line $R$ which has the property that for every $\beta>0$ there exists $T_{\beta}>0$ such that whenever $|\lambda| \leqq T_{\beta}$ and $-\infty<s \leqq t<\infty$,

(1.1) $E \exp \left(\lambda\left[\left(X_{t}-X_{t}\right)-E\left(X_{t}-X_{t}\right)\right]\right) \leqq \exp (\beta|\lambda| \mu(s, t])$.

Then for every function $f \in L_{1}(\mu) \cap L_{\infty}(\mu)$ (over $\left.R\right)$ for which $\|f\|_{1} \leqq 1$, the random variable $\int f(t) d\left[X_{t}-E X_{t}\right]$ is well defined as a limit-in-themean of order 2, and for every $\epsilon>0$ there exists a positive number $\rho<1$ (depending only on $\epsilon$ ) such that

$$
P\left[\left|\int f(t) d\left[X_{t}-E X_{t}\right]\right|>\epsilon\right] \leqq 2 \rho^{1 /\|f\|_{\infty}} .
$$

If $f(s, t)$ is a real-valued function on $R \times R$ such that

$$
f(s, \cdot) \in L_{1}(\mu) \cap L_{\infty}(\mu),\|f(s, \cdot)\|_{1} \leqq 1 \text { and }\|f(s, \cdot)\|_{\infty}=1 / \gamma(s)
$$

then if the stochastic process $\left\{Y_{\mathrm{a}}:-\infty<s<\infty\right\}$ is defined by

$$
Y_{\mathbf{s}}=\int f(s, t) d\left[X_{t}-E X_{t}\right]
$$

it will follow as an immediate consequence of the Theorem that for every $\epsilon>0$ there exists $0<\rho<1$ such that

$$
P\left[\left|Y_{\bullet}\right|>\epsilon\right] \leqq 2 \rho^{\gamma(s)} .
$$

Thus, our Theorem provides a useful probability bound for a large class of stochastic processes derived from processes with independent increments. In particular, if $s^{*}$ is held fixed and $\chi_{0}$ denotes the set characteristic function of the interval $\left(s^{*}, s\right]$, then taking

Received by the editors November 13, 1964.

1 Part of this work was carried out while the authors were at Sandia Corporation and was supported by the United States Atomic Energy Commission. 


$$
f(s, t)=\frac{\chi_{s}(t)}{\mu\left(s^{*}, s\right]}
$$

we obtain

$$
P\left[\left|\left(X_{s}-X_{\iota^{*}}\right)-E\left(X_{\varepsilon}-X_{s^{*}}\right)\right|>\epsilon \mu\left(s^{*}, s\right]\right] \leqq 2 \rho^{\mu\left(z^{*}, s\right]} .
$$

If $\mu\left(s^{*}, s\right] \rightarrow \infty$ as $s \rightarrow \infty,(1.3)$ yields a bound on the rate at which the increments of the process converge to their expectations.

We will show in $\$ 3$ that our Theorem is applicable to the Wiener and Poisson processes and will derive the appropriate versions of (1.3). We will also indicate how the Theorem implies a convergence rate theorem for a generalized version of the law of large numbers for independent random variables first given in [3].

2. Proof of the theorem. The theorem will be proved in two parts. First, we will establish the existence of $\int f(t) d\left[X_{t}-E X_{t}\right]$ as the limitin-the-mean of certain "natural" approximating sums. Inequality 1.2 will be derived in the second part of the proof.

Part 1 of proof. We first establish the following lemma.

LEMMA. Let $X$ be a random variable with $E X=0$ and with the property that for every $\beta>0$ there exists $T_{\beta}>0$ such that for $|\lambda| \leqq T_{\beta}$,

$$
E e^{\lambda x} \leqq e^{\beta R|\lambda|}
$$

Then there exists a number $K_{\beta}>0$, depending on $\beta$ and $T_{\beta}$ but not otherwise on the distribution of $X$, such that

$$
\lambda^{2} E X^{2} \leqq K_{\beta} R|\lambda|, \text { for }|\lambda| \leqq T_{\beta} .
$$

Proof. From (2.1) it follows that for every $\beta>0$ there exists $\epsilon_{\beta}>0$ such that for $|\lambda| \leqq T_{\beta}$,

$$
E e^{\lambda x} \leqq 1+\left(\beta+\epsilon_{\beta}\right)|\lambda| R .
$$

Then, $E\left(e^{\lambda x}+e^{-\lambda x}\right) \leqq 2+2\left(\beta+\epsilon_{\beta}\right)|\lambda| R$ for $|\lambda| \leqq T_{\beta}$ and, since

$$
e^{\lambda x}+e^{-\lambda x}=\sum_{k=0}^{\infty} \frac{(\lambda x)^{2 k}}{(2 k) !} \geqq 2+\frac{(\lambda x)^{2}}{2},
$$

it follows that

$$
\lambda^{2} E X^{2} \leqq 4\left(\beta+\epsilon_{\beta}\right) R|\lambda|,
$$

as was to be shown.

Without loss of generality we will assume $E\left(X_{t}-X_{s}\right)=0$ for all $-\infty<s \leqq t<\infty$. Suppose $f \in L_{1}(\mu) \cap L_{\infty}(\mu)$ and $\|f\|_{1} \leqq 1$. Then there 
exists a sequence of step functions $f_{n}=\sum_{i} c_{n i} \chi_{n i}$ such that $\left\|f_{n}-f\right\|_{1}$ $\rightarrow 0,\left\|f_{n}\right\|_{1} \leqq 1$ and $\sup _{i}\left|c_{n i}\right| \leqq\|f\|_{\infty}$ for all $n$. Here, $\chi_{n i}$ is the set characteristic function of $\Delta_{n i}=\left(a_{n, i-1}, a_{n, i}\right]$, where $-\infty=a_{n,-\infty}<\cdots$ $<a_{n, \theta}<a_{n, 1}<\cdots<a_{n, \infty}=\infty$, and $c_{n i}$ is the value of $f_{n}$ on $\Delta_{n i}$. All but a finite number of the $c_{n i}$ can be taken equal to zero for each $n$.

If $\Delta=(a, b]$, let $X(\Delta)=X_{b}-X_{a}$. For each $n$ form the stochastic integral

$$
Y_{n}=\int f_{n}(t) d X_{t}=\sum c_{n i} X\left(\Delta_{n i}\right)
$$

We will show that the sequence $\left\langle Y_{n}\right\rangle$ is a Cauchy sequence in the stochastic Lebesgue space $\mathfrak{L}_{2}$.

Fix $m$ and $n$ and let $\Delta_{i}$ be the element with index $i$ of the partition obtained by ordering the merged partition $\left\{\Delta_{m i} \cap \Delta_{n j}:-\infty \leqq i, j \leqq \infty\right\}$. Let $c_{i}^{(m)}$ and $c_{i}^{(n)}$ be the values of $f_{m}$ and $f_{n}$ respectively on $\Delta_{i}$. Then, by the independent increments assumption

$$
E\left(Y_{m}-Y_{n}\right)^{2}=\sum_{i}\left(c_{i}^{(m)}-c_{i}^{(n)}\right)^{2} E X^{2}\left(\Delta_{i}\right)
$$

Let $r=2\|f\|_{\infty}$, and let

$$
\lambda_{i}=\frac{c_{i}^{(m)}-c_{i}^{(n)}}{r} T_{\beta} \text { for fixed } \beta>0 .
$$

Then, by Condition 1.1 and the lemma,

$$
\begin{aligned}
E\left(Y_{m}-Y_{n}\right)^{2} & =\frac{r^{2}}{T_{\beta}^{2}} \sum_{i} \lambda_{i}^{2} E X^{2}\left(\Delta_{i}\right) \\
& \leqq \frac{K_{\beta} r^{2}}{T_{\beta}^{2}} \sum_{i}\left|\lambda_{i}\right| \mu\left(\Delta_{i}\right) \\
& \leqq \frac{K_{\beta} r}{T_{\beta}} \sum_{i}\left|c_{i}^{(m)}-c_{i}^{(n)}\right| \mu\left(\Delta_{i}\right) \\
& \leqq \frac{2 K_{\beta}\|f\|_{\infty}}{T_{\beta}}\left\|f_{m}-f_{n}\right\|_{1 .}
\end{aligned}
$$

Then, $\left\langle Y_{n}\right\rangle$ is Cauchy in $\mathscr{L}_{2}$ which implies the existence of $Y \in \mathscr{L}_{2}$ such that $E\left(Y-Y_{n}\right)^{2} \rightarrow 0$. It is easily shown that $Y$ is independent of the sequence $\left\langle f_{n}\right\rangle$ tending to $f$, so it is proper to write $Y=\int f(t) d X_{t}$ (see, e.g., [2]). 
Part 2 of proof. Fix $\epsilon>0$. By a well-known inequality [4, p. 157], for all $\lambda \geqq 0$,

$$
\begin{aligned}
P\left[ \pm Y_{n}>\epsilon\right] & =P\left[ \pm \sum_{i} c_{n i} X\left(\Delta_{n i}\right)>\epsilon\right] \\
& \leqq E \exp \left\{ \pm \lambda \sum_{i} c_{n i} X\left(\Delta_{n i}\right)-\lambda \epsilon\right\} \\
& =\sigma^{-\lambda i} \prod_{i} E \exp \left[ \pm \lambda c_{n i} X\left(\Delta_{n i}\right)\right] .
\end{aligned}
$$

$\operatorname{Set} \beta=\epsilon / 2$ and $\lambda=T_{\beta} /\|f\|_{\infty}$. Then, $\left|\lambda c_{n i}\right| \leqq T_{\beta}$ and from Condition 1.1,

$$
\begin{aligned}
P\left[ \pm Y_{n}>\epsilon\right] & \leqq e^{-\lambda \epsilon} \exp \left\{\beta|\lambda|\left\|f_{n}\right\|_{1}\right\} \\
& \leqq\left(\exp \left(-T_{\beta \epsilon} / 2\right)\right)^{1 /\|||\|_{\infty} .}
\end{aligned}
$$

Inequality (1.2) now follows with $\rho=\exp \left(-T_{\beta} \epsilon / 2\right)$ because the $\mathfrak{L}_{2}$ convergence of $\left\langle Y_{n}\right\rangle$ implies $P\left[ \pm Y_{n}>\epsilon\right] \rightarrow P\left[ \pm \int f(t) d X_{t}>\epsilon\right]$ and because of the inequality $P[|X|>\epsilon] \leqq P[X>\epsilon]+P[-X>\epsilon]$.

3. Applications of the Theorem. 1. If $X$ is the Wiener process, $X_{t}: N\left(0, \sigma^{2} t\right)$, Condition (1.1) is satisfied with $\mu=\sigma^{2} \times$ Lebesgue measure and $T_{\beta}=2 \beta$. Then, for $0 \leqq s<t<\infty$, Inequality (1.3) becomes

$$
P\left[\left|X_{t}-X_{\bullet}\right| \geqq \sigma^{2}(t-s) \epsilon\right] \leqq 2 \rho^{\sigma^{2}(t-s)} .
$$

This is comparable with the inequality given in $[2$, p. 392].

2. If $X$ is a Poisson process, $X_{t}-X_{s}: \odot\left(\mu_{s, t}\right)$, where $\mu_{s, t}=\mu(s, t]$ for a $\sigma$-finite measure $\mu$, then Condition (1.1) is satisfied with this measure and $T_{\beta}$ the root of largest modulus of the equation $e^{\lambda}-\lambda-1=\beta|\lambda|$. It follows from Inequality (1.3) that for $-\infty<s<t<\infty$

$$
P\left[\left|\left(X_{t}-X_{s}\right)-\mu_{s, t}\right|>\epsilon \mu_{s, t}\right] \leqq 2 p^{\mu, t} .
$$

3. Let $\mu$ be counting measure on the integers and let $Y_{n}=X_{n}$ $-X_{n-1}$. If $f \in L_{1}(\mu) \cap L_{\infty}(\mu)$ is the doubly infinite sequence $\left\langle a_{k}\right\rangle_{k=-\infty}^{\infty}$ then the following is an immediate corollary of our Theorem.

COROLLARY. Let $\left\langle Y_{n}\right\rangle_{n--\infty}^{\infty}$ be an independent sequence of random variables such that $E Y_{n}=0$ all $n$, and for every $\beta>0$ there exists $T_{\beta}>0$ such that for $|\lambda| \leqq T_{\beta}$,

$$
E \exp \left(\lambda Y_{n}\right) \leqq \exp (\beta|\lambda|) \text { uniformly in } n \text {. }
$$

Let $\left\langle a_{k}\right\rangle_{k--\infty}^{\infty}$ be a sequence of real constants such that

(i) $\sum_{k=-\infty}^{\infty}\left|a_{k}\right| \leqq 1$, 
(ii) $\max _{k}\left|a_{k}\right|=M<\infty$.

Then, $S=\sum_{k--\infty}^{\infty} a_{k} Y_{k}$ is well defined as a limit-in-the-mean of order 2 of its partial sums and for every $\epsilon>0$ there exists a positive number $\rho<1$ such that

$$
P[|S|>\epsilon] \leqq 2 \rho^{1 / M} .
$$

This theorem generalizes a convergence rate result for the law of large numbers originally established by Cramér [1].

A slightly stronger version of this theorem was proved in [3].

\section{REFERENCES}

1. H. Cramer, Sur un nouveau theoreme-limite de la theorie des probabilites, Actualités Sci. Ind., No. 736, Paris, 1938.

2. J. L. Doob, Stochastic processes, Wiley, New York, 1952.

3. D. L. Hanson and L. H. Koopmans, On the convergence rate of the law of large numbers for linear combinations of independent random variables, Ann. Math. Statist. 36 (1965), 559-564.

4. M. Loeve, Probability theory, 2nd ed., Van Nostrand, New York, 1960.

UNIVERSITY OF MISSOURI AND UNIVERSITY OF NEW MEXICO 